\title{
Robust asymptotic entanglement under multipartite collective dephasing
}

\author{
Edoardo G. Carnio, ${ }^{1,2, *}$ Andreas Buchleitner, ${ }^{1,3}$ and Manuel Gessner ${ }^{1, \dagger}$ \\ ${ }^{1}$ Physikalisches Institut, Albert-Ludwigs-Universität Freiburg, Hermann-Herder-Straße 3, 79104 Freiburg, Germany \\ ${ }^{2}$ Department of Physics, University of Warwick, Coventry, CV4 7AL, United Kingdom \\ ${ }^{3}$ Freiburg Institute for Advanced Studies, Albert-Ludwigs-Universität Freiburg, Albertstraße 19, 79104 Freiburg, Germany
}

(Dated: October 4, 2018)

\begin{abstract}
We derive an analytic solution for the ensemble-averaged collective dephasing dynamics of $N$ noninteracting atoms in a fluctuating homogeneous external field. The obtained Kraus map is used to specify families of states whose entanglement properties are preserved at all times under arbitrary field orientations, even for states undergoing incoherent evolution. Our results apply to arbitrary spectral distributions of the field fluctuations.
\end{abstract}

PACS numbers: 03.65.Yz, 03.65.Ud, 03.67.Mn

Control of the coherent evolution of quantum systems in noisy environments [1] is one of the crucial prerequisites for exploiting nontrivial quantum effects in composite systems of increasing complexity. Whether in the context of controlled molecular reactions [2], of many-particle quantum dynamics [3], or of quantum computers and simulators [4], uncontrolled fluctuations and noise are detrimental to most purposes of optimal control. Various strategies may be followed to counteract the harmful influence of the environment: shielding the system degrees of freedom [5], correcting environmentinduced errors [6], exploiting basins of attraction in dissipative systems [8], or compensating dissipation-e.g., by coherent dynamics [7], dynamical decoupling [9], or periodic measurements [10]. Such approaches can effectively reduce the environmental effects and can enhance coherence times, but a perfect protection of the quantities of interest is generally not possible.

By restricting to superposition states within a decoherencefree subspace, initially entangled states can be shielded completely from collective noise sources, hence protecting their entanglement at all times [11, 12]. However, such subspaces are rather fragile to small perturbations, which limit their applicability in the context of dynamical processes [13]. Here, we identify conditions that ensure complete preservation of arbitrary degrees of entanglement, even for states that are not invariant under an incoherent time evolution. Specifically, we consider an important class of environment-induced fluctuations, which are frequently encountered in state-of-the-art experiments [14-16]: they manifest in intensity fluctuations of spatially homogeneous experimental control fields, giving rise to an effective dephasing process. We show how control of the external field's orientation can lead to the complete preservation of entanglement in bipartite-as well as multipartitesettings, for arbitrary spectral characteristics of the control field fluctuations. We further identify families of states exhibiting time-invariant entanglement for arbitrary orientations of the external field.

To set the stage, let us consider a collection of $N$ noninteracting atomic two-level systems with identical energy splitting $\hbar \omega$ controlled, e.g., by a homogeneous magnetic field. Integration over the unavoidable fluctuations of the latter's strength will induce a probability distribution $p(\omega)$ of the characteristic energy splitting, and the $N$-atom quantum state at time $t$ therefore needs to be described by the statistical operator

$$
\rho(t)=\int p(\omega) U_{\omega}(t)^{\otimes N} \rho(0) U_{\omega}^{\dagger}(t)^{\otimes N} \mathrm{~d} \omega,
$$

provided the field fluctuations occur on time scales which are longer than the time $t$ over which the $N$-atom state is propagated by the unitary $U_{\omega}(t)^{\otimes N}$. In order to assess the open system time evolution of the quantum correlations inscribed into the $\mathrm{N}$-atom system, it is convenient to derive an explicit expression for $\rho(t)$ in terms of the spectral distribution $p(\omega)$ characterizing the fluctuations.

The single-atom propagator $U_{\omega}(t)=\mathrm{e}^{-\mathrm{i} H_{\omega} t / \hbar}$ is generated by the time-independent single-atom Hamiltonian $H_{\omega}=$ $(\hbar \omega / 2) \boldsymbol{n} \cdot \boldsymbol{\sigma}$, with $\boldsymbol{\sigma}=\left(\sigma_{x}, \sigma_{y}, \sigma_{z}\right)$ being the vector of the Pauli matrices and $\boldsymbol{n}$ the orientation of the field. $H_{\omega}$ describes atomic dipoles interacting with electromagnetic fields, as, e.g., the electronic qubits in trapped-ion quantum registers [14, 17]. Introducing pairs of orthogonal projectors $\Lambda_{ \pm}=\left(\mathbb{I}_{2} \pm \boldsymbol{n} \cdot \boldsymbol{\sigma}\right) / 2$, we can rewrite the time evolution operator for a collection of $N$ atoms as

$$
\begin{aligned}
U_{\omega}(t)^{\otimes N} & =\left(\mathrm{e}^{-\mathrm{i} \omega t / 2} \Lambda_{+}+\mathrm{e}^{\mathrm{i} \omega t / 2} \Lambda_{-}\right)^{\otimes N} \\
& =\sum_{j=0}^{N} \mathrm{e}^{\mathrm{i} \omega t(j-N / 2)} \Theta_{j},
\end{aligned}
$$

where we have defined the operators

$$
\Theta_{j}=\frac{1}{j !(N-j) !} \sum_{s \in \Sigma_{N}} V_{s}\left[\Lambda_{-}^{\otimes j} \otimes \Lambda_{+}^{\otimes N-j}\right] V_{s}^{\dagger},
$$

where $\Sigma_{N}$ denotes the symmetric group and $V_{s}=$ $\sum_{i_{1} \ldots i_{N}}\left|i_{s(1)} \ldots i_{s(N)}\right\rangle\left\langle i_{1} \ldots i_{N}\right|$ represents the permutation $s \in$ $\Sigma_{N}$ in the operator space of $N$ qubits. The ensemble-averaged state after time $t$,

$$
\rho(t)=\sum_{j, k=0}^{N} M_{j k}(t) \Theta_{j} \rho(0) \Theta_{k},
$$

is then fully characterized by the Toeplitz matrix $M(t)$, whose elements $M_{j k}(t)=\varphi[(j-k) t]$ are generated by the characteristic function $\varphi(t)=\int p(\omega) \mathrm{e}^{\mathrm{i} \omega t} \mathrm{~d} \omega$ of the probability distribution $p(\omega)$. Bochner's theorem [18] ensures that $M(t)$ is a 
Hermitian semipositive definite matrix for all $t$. Diagonalization leads to the canonical Kraus form [19]

$$
\rho(t)=\epsilon_{t, 0}[\rho(0)]=\sum_{i=0}^{N} A_{i}(t) \rho(0) A_{i}^{\dagger}(t),
$$

where the Kraus operators $A_{i}(t)=\sum_{j=0}^{N} \sqrt{\lambda^{i}(t)} \lambda_{j}^{i}(t) \Theta_{j}$ contain the eigenvalues $\lambda^{i}(t)$ and the components of the eigenvectors $\lambda^{i}(t)$ of $M(t)$. Note, from the structure of $A_{i}(t)$, that the above defined Kraus operators mediate an effective interaction between the individual qubits-with its origin in the spatial homogeneity of the external field. These environment-induced interactions are able to create discord-type quantum correlations [17] and, as we will show in this Letter, given the appropriate control of $\boldsymbol{n}$, can uphold multipartite entanglement at all times for arbitrary intensity fluctuations.

Using the fact that the operators $\Lambda_{ \pm}$are orthogonal projectors in $\mathbb{C}^{2}$, we can immediately show that both of the operators $\left\{\Theta_{j}\right\}_{j}$ and $\left\{A_{i}(t)\right\}_{i}$ satisfy the condition $\sum_{i} A_{i}^{\dagger}(t) A_{i}(t)=$ $\sum_{i} \Theta_{i}^{\dagger} \Theta_{i}=\mathbb{I}_{2}^{N}$, which ensures that the map $\epsilon_{t, 0}$ defined in (5) from now on called the "collective dephasing" map-is not only completely positive but also trace preserving for all $t$ $[19,20]$.

For absolutely continuous distribution functions [24], the characteristic function vanishes asymptotically; i.e., $\lim _{t \rightarrow \infty} \varphi(t)=0$. We then have that $\lim _{t \rightarrow \infty} M(t)=\mathbb{I}_{N+1}$, and thus the Kraus operators reduce to $\lim _{t \rightarrow \infty} A_{i}(t)=\Theta_{i}$. The asymptotic $N$-qubit state is thus given by $\rho_{s}=\lim _{t \rightarrow \infty} \rho(t)=$ $\sum_{i} \Theta_{i} \rho(0) \Theta_{i}$. Because the operators $\Theta_{i}$ depend exclusively on the magnetic field direction $\boldsymbol{n}$, the latter completely determines the properties of the asymptotic state.

To gain some intuition on the time evolution of the entanglement properties, e.g., of an $\mathrm{N}$-ion quantum register under the action of the collective dephasing map, we first consider two-qubit states with maximally mixed reduced density matrices (also called Bell-diagonal states). Such states allow for a simple geometric representation, since they are fully characterized by the matrix $\beta_{i j}=\operatorname{tr}\left(\rho \cdot \sigma_{i} \otimes \sigma_{j}\right)$ [1]. There always exist unitary operations $U_{A}$ and $U_{B}$ such that $U_{A} \rho U_{B}^{\dagger}$ has a diagonal $\beta$ matrix, $\beta=\operatorname{diag}\left(d_{1}, d_{2}, d_{3}\right)$, while $\rho$ and $U_{A} \rho U_{B}^{\dagger}$ have the same separability properties [27]. This allows us to associate with each density matrix a point $\boldsymbol{d}=\left(d_{1}, d_{2}, d_{3}\right)^{T} \in \mathbb{R}^{3}$ and, because of positivity, any such point must lie inside a tetrahedron [Fig. 1 (a)] of vertices $(-1,-1,-1)^{T},(-1,1,1)^{T},(1,-1,1)^{T}$ and $(1,1,-1)^{T}$, which represent the four Bell states [1]. Inside this tetrahedron we distinguish an inner octahedron, which contains the separable states, from the four remaining corners, which consist of the entangled states [1] and are labeled by the Bell state they contain (e.g., $\left|\Psi_{-}\right\rangle$-corner). In this setting Wootters's concurrence [2] is simply the distance from the faces of the octahedron: $C(\boldsymbol{d})=1 / 2 \max \left\{0, \sum_{i}\left|d_{i}\right|-1\right\}$. Equidistant points, parallel to the surfaces of the octahedron, form the "isoconcurrence" planes.

In the tetrahedron, the collective dephasing evolution is always constrained onto a plane defined by $\operatorname{Tr} \beta(t)=\operatorname{Tr} \beta(0)$
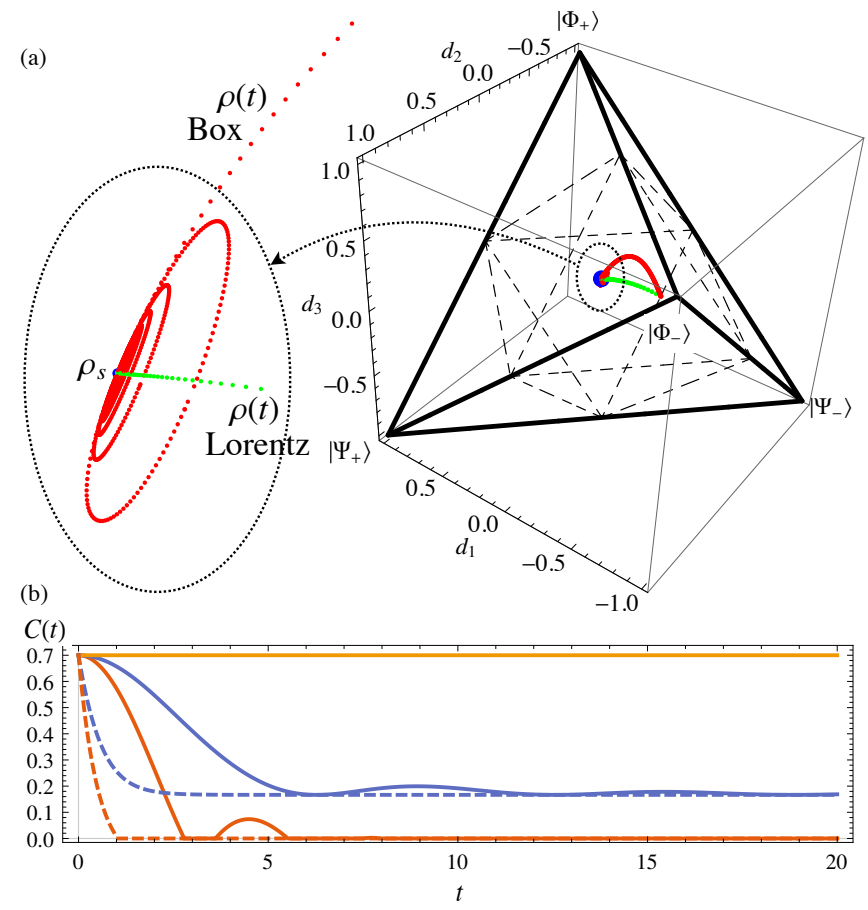

FIG. 1. Dynamics of a bipartite system with the initial state $\rho_{0}=\mathbb{I}_{4} / 20+4\left|\Phi^{-}\right\rangle\left\langle\Phi^{-}\right| / 5$. (a) The evolution of the state under Lorentzian- (green) and box-distributed (red) noise, $C_{0,1}(\omega)$ and $B_{0,1}(\omega)$ (see the text for a definition), respectively, with a magnetic field direction $\boldsymbol{n}=(2,1,1)^{T} / \sqrt{6}$, is depicted inside the tetrahedron of Bell-diagonal states. The inner octahedron marks the set of separable states. (b) Depending on the orientation of the magnetic field, the concurrence remains constant [for $\boldsymbol{n}=(1,0,0)^{T}$, orange], decays to a finite value [for $\boldsymbol{n}=(2,1,1)^{T} / \sqrt{6}$, blue], or decays to zero (for $\boldsymbol{n}=(0,1,1)^{T} / \sqrt{2}$, red). The decay is monotonic for Lorentzian (dashed lines), or nonmonotonic for box-distributed (solid lines) noise.

[20]. In the $\left|\Psi_{-}\right\rangle$-corner, these planes coincide with isoconcurrence planes, which implies that entanglement is preserved for all of these states, for arbitrary directions of the magnetic field. This leads to a finite-measure set of states with timeinvariant concurrence, despite the fact that those states do evolve in time, $\rho(t) \neq \rho(0)$ [29]. For entangled states outside the $\left|\Psi_{-}\right\rangle$-corner, we can use Eq. (4) to predict the final concurrence as $C_{\boldsymbol{d}, \mathrm{f}}(\boldsymbol{n})=1 / 2 \max \left\{0, \sum_{i=1}^{3}\left(1-2 n_{i}^{2}\right) d_{i}-1\right\}$, where $\boldsymbol{n}=\left(n_{1}, n_{2}, n_{3}\right)^{T}$ and $\boldsymbol{d}=\left(d_{1}, d_{2}, d_{3}\right)^{T}$ characterizes the initial state [20]. Thus, by solving for $\boldsymbol{n}$, we can always find a field direction such that the entanglement is preserved at all times. This can be seen from the long-time limit in Fig. 1 (b), whereas the transient time evolution depends on $p(\omega)$, as we will discuss later.

Collective interactions become particularly relevant in multipartite settings, where decoherence and dissipation can be strongly enhanced [11,33-35]. To analyze the effect of collective dephasing on multipartite entanglement, analytic expressions à la Wootters [2] are not available. Intricate hierarchies of multipartite entanglement [36] can, however, be characterized efficiently by resorting to separability criteria 


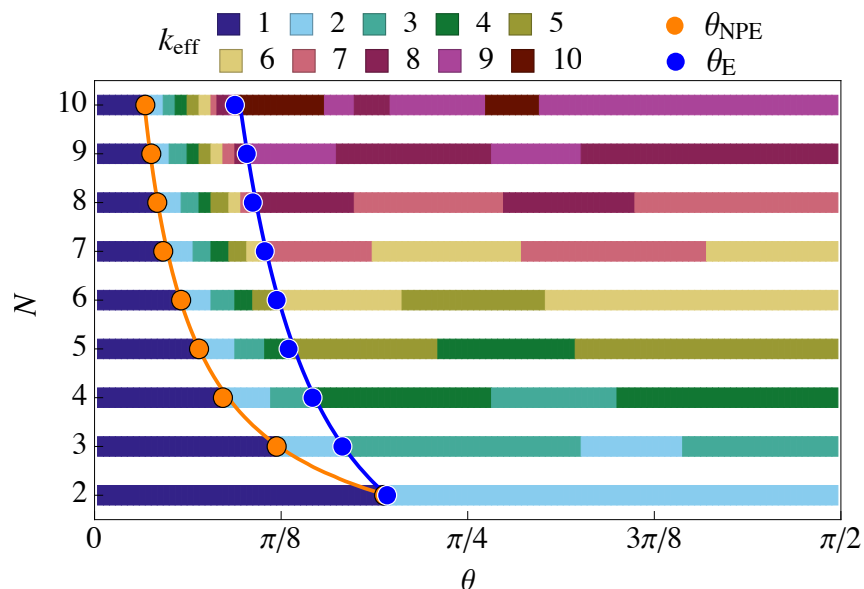

FIG. 2. Influence of collective dephasing (5) on $N$-partite entangled $W$-states. Here we show the upper bound $k_{\text {eff }}$ to the asymptotic state's separability vs the polar angles $0 \leq \theta \leq \pi / 2$ (measured from the $z$ axis to the $x, y$-plane) of the fluctuating magnetic field's direction. In blue and orange we show, respectively, the dependence of the critical angles $\theta_{\mathrm{E}}$ and $\theta_{\mathrm{NPE}}$, Eqs. (6) and (7), on $N$. The dots correspond to the numerical estimation of the smallest angle where $k_{\text {eff }}$ changes from 1 to 2 (defining $\theta_{\mathrm{NPE}}$ ) or from $N-1$ to $N$ (defining $\theta_{\mathrm{E}}$ ). The uncertainty on this angle, due to the finite bin width of our sampling, is covered by the dot size. The lines represent the expressions (6) and (7).

based on inequalities [37,38]. An $N$-partite state $\rho$ is called $k$ separable if it can be written as a mixture of states of the form $\rho=\rho_{A_{1}} \otimes \cdots \otimes \rho_{A_{k}}$, where $A_{1} \ldots A_{k}$ label a division of the $N$ parties into $k$ subgroups. For instance, the matrix elements in an arbitrary basis of any $k$-separable $N$-qubit density matrix $\rho$ satisfy $\sum_{0 \leq i<j \leq N-1}\left|\rho_{2^{i}+1,2^{j}+1}\right| \leq \sum_{0 \leq i<j \leq N-1} \sqrt{\rho_{1,1} \rho_{2^{i}+2^{j}+1,2^{i}+2^{j}+1}}+$ $(N-k) / 2 \sum_{i=0}^{N-1} \rho_{2^{i}+1,2^{i}+1}[39,40]$. Defining $k_{\text {eff }}$ as the largest integer $k$ saturating this inequality provides an upper bound to the states $k$-separability class, as $k \leq k_{\text {eff }}$. When $k_{\text {eff }}<2$ the state certainly contains genuine multipartite entanglement, i.e., it is not even 2-separable, while the state can be fully separable ( $N$-separability) only if $k_{\text {eff }} \geq N$.

We consider the initial ( $N$-partite entangled) $W$-state, $|W\rangle=$ $(|10 \ldots 0\rangle+|01 \ldots 0\rangle+\cdots+|0 \ldots 01\rangle) / \sqrt{N}$, where $|1\rangle$ and $|0\rangle$ denote eigenstates of $\sigma_{z}$. Since the collective dephasing map (5) is invariant under the operation $\boldsymbol{n} \rightarrow-\boldsymbol{n}$ and, additionally, this class of states exhibits rotational symmetry around the $z$ axis, the polar angle $\theta \in[0, \pi / 2]$ between $\boldsymbol{n}$ and the $z$-axis (which is defined by the local eigenbasis of the initial state) fully determines the evolution of the state under (5). Figure 2 displays the entanglement properties of the resulting asymptotic state, characterized by $k_{\mathrm{eff}}$ as a function of $\theta$. In general, there are relatively small angle intervals that lead to a fully separable state, and typically $k_{\text {eff }}$ shows nonmonotonic dependence on $\theta$.

Our numerical data (Fig. 2) suggest that the asymptotic state resulting from $|W\rangle$ is certainly entangled (i.e. $k_{\text {eff }}<N$ ) as long as $\theta<\theta_{\mathrm{E}}$, where

$$
\theta_{\mathrm{E}}(N)=\arctan (1 / \sqrt{N}) .
$$

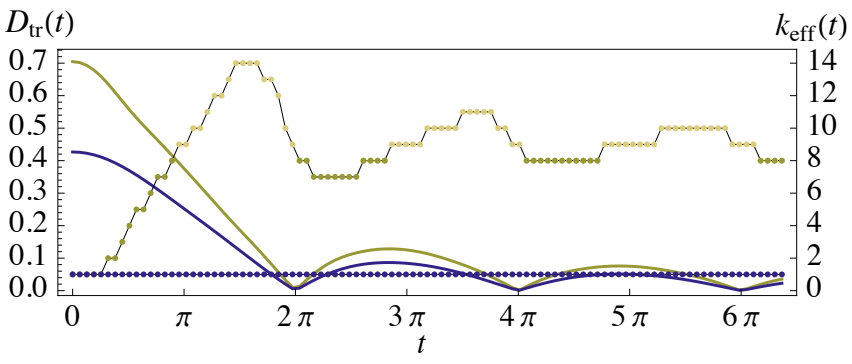

FIG. 3. (Color online) Evolution of the trace distance $D_{\text {tr }}$ between $\rho(t)$ and the asymptotic state $\rho_{s}$ (solid lines), and of the state's separability bound $k_{\text {eff }}$ (connected dots), respectively, for eight-partite $|W\rangle$ (green) and $|\tilde{W}\rangle$ (blue) initial states (see the text for a definition), boxdistributed noise fluctuations $B_{0,1}(\omega)$, and a polar angle $\theta=\pi / 8$. The values where $k_{\text {eff }}>N$, marked in a lighter green shade, indicate that the state is compatible with full separability, and hence they do not provide additional information than the case $k_{\text {eff }}=N$. Note that the $|\tilde{W}\rangle$ state exhibits time-invariant genuine multipartite entanglement outside of a time-invariant subspace.

Conversely, when we choose a magnetic field that is close to the $z$-direction, the initial $N$-partite entanglement of the $W$ state will be preserved during the dephasing process, since $|W\rangle$ is part of an eigenspace of the Hamiltonian for $\boldsymbol{n}=(0,0,1)^{T}$. Again, we find a critical angle

$$
\theta_{\mathrm{NPE}}(N)=\arctan (1 / \sqrt{N(N-1)}),
$$

such that for $\theta<\theta_{\mathrm{NPE}}$, the asymptotic state will contain genuine multipartite entanglement $\left(k_{\mathrm{eff}}<2\right)$. Conditions (6) and (7) provide a finite range of orientations that ensure preservation of entanglement properties in initial $W$-states. However, as the number of qubits gets larger, higher accuracy is required to maintain $N$-partite entanglement $\left(\theta_{\mathrm{NPE}}\right)$ or at least some type of entanglement $\left(\theta_{\mathrm{E}}\right)$. Moreover, the fast decay of $\theta_{\mathrm{NPE}}$ with the number of qubits confirms that genuine $\mathrm{N}$ partite entanglement is much more fragile than bipartite entanglement $[36,41]$, which is able to resist a larger range of field directions. We remark here that in order to modify $\theta$ in a trapped-ion experiment it is much more natural to apply unitary pulses to the initial state to shift its relative orientation to the field, instead of actually changing the orientation of the external field [17].

Furthermore, we notice that states displaying time-invariant entanglement properties can be found in the multipartite case, too. One example is given by a specific family of $W$ states, whose single-excited states carry the relative phases $\left\{\mathrm{e}^{\mathrm{i} 2 \pi k / N}\right\}_{k=1}^{N}$ in an arbitrary order, e.g.,

$$
\begin{aligned}
|\tilde{W}\rangle= & \left(\mathrm{e}^{\mathrm{i}(2 \pi / N)}|10 \ldots 0\rangle+\mathrm{e}^{\mathrm{i}(4 \pi / N)}|01 \ldots 0\rangle\right. \\
& +\cdots+|0 \ldots 01\rangle) / \sqrt{N}
\end{aligned}
$$

As shown in Fig. 3, this state remains $N$-partite entangled throughout the whole evolution, but the state itself evolves into a stationary state, as is displayed by the trace distance $D_{\text {tr }}(t)=\left\|\rho(t)-\rho_{s}\right\| / 2$, where $\|X\|=\operatorname{Tr} \sqrt{X^{\dagger} X}$ denotes the 
trace norm. The question remains whether this state is part of a finite-measure set of states whose multipartite entanglement properties are conserved, similarly to the $\left|\Psi_{-}\right\rangle$-corner in the bipartite case-notice that $|\tilde{W}\rangle$ reduces to $\left|\Psi_{-}\right\rangle$when $N=2$. Such states would constitute ideal candidates for quantum computations by exhibiting invariance under collective dephasing effects.

Let us finally characterize a family of time-invariant states, for arbitrarily many qubits. Using Eq. (4), it can be shown that any state of the form $\rho_{W}=\sum_{s \in \Sigma_{N}} c_{s} V_{s}$, where $c_{s}$ are arbitrary coefficients and $V_{s}$ are the permutation operators defined above, satisfies $\rho(t)=\rho(0)$ at all times [20]. These states, known as multipartite Werner states [3], are also characterized by their invariance under arbitrary local unitary transformations $U^{\otimes N}$ [42]. Since such transformations describe collective changes of the local qubit coordinate systems, it is quite intuitive that these states are time-invariant for arbitrary directions of the external field. This identifies a $(N !-1)$-parameter family of states that always span a decoherence-free subspace $[11,12]$. In the geometric picture of Fig. 1 (a), these states lie on the line passing through the origin of the tetrahedron and the $\left|\Psi_{-}\right\rangle$-state.

We conclude with some remarks on the transient evolution towards the asymptotic state. To determine how close the evolved state is to its asymptotic state, we again employ the trace distance $D_{\mathrm{tr}}(t)$ which has a clear interpretation in terms of the distinguishability of the quantum states [43]. In our present context, the trace distance is employed as an autocorrelation function, which reveals the monotonicity of the quantum evolution.

While different types of noise fluctuations lead to the same asymptotic state, as discussed earlier, the transient behavior can be qualitatively different, as displayed in Fig. 1. When the distribution $p(\omega)$ is Lorentzian, $C_{\omega_{0}, \gamma}(\omega)=(\gamma / \pi)\left[\left(\omega-\omega_{0}\right)^{2}+\right.$ $\left.\gamma^{2}\right]^{-1}$, or Gaussian, $N_{\omega_{0}, \sigma}(x)=\exp \left(-\left(\omega-\omega_{0}\right)^{2} / 2 \sigma^{2}\right) / \sqrt{2 \pi \sigma^{2}}$ (as suggested in Ref. [41]), the properties of the state, such as the concurrence, decay exponentially towards their asymptotic value [Fig. 1 (b)]. When we instead consider the box distribution over the interval $\left[0, \omega_{0}\right]$, i.e., $B_{0, \omega_{0}}(\omega)=$ $\left[\Theta_{H}(\omega)-\Theta_{H}\left(\omega-\omega_{0}\right)\right] / \omega_{0}$, where $\Theta_{H}(\omega)$ is the Heaviside step function, we observe a nonmonotonic approach of the quantum system to the asymptotic state (Fig. 1). In fact, for this distribution the characteristic function $\varphi(t)$ is proportional to $\sin \left(\omega_{0} t\right) / t$, which asymptotically decreases on a significantly longer time scale than the exponential decay characterizing the Lorentzian or Gaussian distributions. This nonmonotonic behavior also implies that the ensemble-averaged dynamics of noninteracting atoms in a fluctuating classical field cannot be modeled by an effective Markovian environment for certain noise distributions $p(\omega)[34,44]$. These frequency fluctuations, therefore, take on the role of the environment's spectral density in a standard open-system description of decoherence [34, 35].

To summarize, we have provided a model for the dephasing dynamics of a collection of noninteracting atoms subject to a homogeneous external field of fluctuating intensity. The effec- tive environment-induced interactions are described analytically by an exact solution in terms of a canonical Kraus map, able to describe the time evolution of multipartite systems under arbitrary intensity fluctuations. Our model applies to a variety of experiments in atomic physics, and describes one of the dominant error sources for state-of-the-art trapped-ion experiments. Complete theoretical control on transient as well as asymptotic dynamics allows for the formulation of precise conditions for preserving relevant quantities, such as entanglement, as well as for the identification of families of states whose properties are completely insensitive to the direction of the external field.

Acknowledgment. M.G. thanks the German National Academic Foundation for their support.

e.carnio@warwick.ac.uk

manuel.gessner@physik.uni-freiburg.de

[1] A. Buchleitner and K. Hornberger (Eds.), Coherent Evolution in Noisy Environments, in Lecture Notes in Physics, Vol. 611 (Springer, Berlin, Germany, 2002).

[2] M. Shapiro and P. Brumer, Quantum Control of Molecular Processes (Wiley, New York, NY, 2012).

[3] F. Platzer, F. Mintert, and A. Buchleitner, Optimal Dynamical Control of Many-Body Entanglement, Phys. Rev. Lett. 105 020501 (2010); F. Lucas, F. Mintert, and A. Buchleitner, Tailoring many-body entanglement through local control, Phys. Rev. A 88, 032306 (2013).

[4] M. Nielsen and I. Chuang, Quantum Computation and Quantum Information (Cambridge University Press, Cambridge, UK, 2000).

[5] S. Haroche, Nobel Lecture: Controlling photons in a box and exploring the quantum to classical boundary, Rev. Mod. Phys. 85, 1083 (2013); D. J. Wineland, Nobel Lecture: Superposition, entanglement, and raising Schrödinger's cat, Rev. Mod. Phys. 85, 1103 (2013).

[6] J. Chiaverini et al., Realization of quantum error correction, $\mathrm{Na}$ ture 432, 602 (2004).

[7] S. Sauer, C. Gneiting, and A. Buchleitner, Optimal Coherent Control to Counteract Dissipation, Phys. Rev. Lett. 111, 030405 (2013); Stabilizing entanglement in the presence of local decay processes, Phys. Rev. A 89, 022327 (2014).

[8] B. Kraus et al., Preparation of entangled states by quantum Markov processes, Phys. Rev. A 78, 042307 (2008).

[9] L. Viola and S. Lloyd, Dynamical suppression of decoherence in two-state quantum systems, Phys. Rev. A 58, 2733 (1998); L. Viola, E. Knill, and S. Lloyd, Dynamical Decoupling of Open Quantum Systems, Phys. Rev. Lett. 82, 2417 (1999); K. Khodjasteh et al., Designing a practical high-fidelity long-time quantum memory, Nature Comm. 4, 2045 (2013).

[10] P. Facchi and S. Pascazio, Quantum Zeno Subspaces, Phys. Rev. Lett. 89, 080401 (2002); F. Schäfer et al., Experimental realization of quantum zeno dynamics, Nature Comm. 5, 3194 (2014).

[11] G. M. Palma, K.-A. Suominen, and A. K. Ekert, Quantum Computers and Dissipation, Proc. R. Soc. Lond. A 452, 567 (1996).

[12] D. A. Lidar, I. L. Chuang, and K. B. Whaley, Decoherence-Free Subspaces for Quantum Computation, Phys. Rev. Lett. 81, 2594 (1998).

[13] D. Bacon, D. A. Lidar, and K. B. Whaley, Robustness of 
decoherence-free subspaces for quantum computation, Phys. Rev. A 60, 1944 (1999).

[14] H. Häffner, C. F. Roos, and R. Blatt, Quantum computing with trapped ions, Phys. Rep. 469, 155 (2008).

[15] C. Gross et al., Nonlinear atom interferometer surpasses classical precision limit, Nature 464, 1165 (2010).

[16] P. Schindler et al., A quantum information processor with trapped ions, New J. Phys. 15, 123012 (2013).

[17] B. P. Lanyon et al., Experimental Generation of Quantum Discord via Noisy Processes, Phys. Rev. Lett. 111, 100504 (2013).

[18] W. Rudin, Fourier Analysis on Groups (John Wiley \& Sons, Inc., Hoboken, NJ, 1990).

[19] I. Bengtsson and K. Życzkowski, Geometry of Quantum States: An Introduction to Quantum Entanglement (Cambridge University Press, Cambridge, UK, 2007).

[20] For further details on the properties of the collective dephasing map see the Supplementary Material, which includes Refs. [13].

[21] R. Horodecki, and M. Horodecki, Information-theoretic aspects of inseparability of mixed states, Phys. Rev. A 54, 1838 (1996).

[22] W. K. Wootters, Entanglement of Formation of an Arbitrary State of Two Qubits, Phys. Rev. Lett. 80, 2245 (1998).

[23] T. Eggeling and R. F. Werner, Separability properties of tripartite states with $U \otimes U \otimes U$ symmetry, Phys. Rev. A 63, 042111 (2001).

[24] These are just the "usual" continuous probability distributions $f$ that can be defined via (Lebesgue-integrable) cumulative distribution functions $F$ as $F(x)=\int_{-\infty}^{x} f(a) \mathrm{d} a$. The RiemannLebesgue lemma [25] then ensures that the characteristic function vanishes asymptotically (see Ref. [26]).

[25] W. Rudin, Real and Complex Analysis (McGraw-Hill, New York, 1987).

[26] E. Lukacs, Characteristic Functions (Griffin, London, UK, 1970).

[27] The $\beta$ matrix can be diagonalized via a transformation $O_{A} \beta O_{B}^{T}$ based on orthogonal matrices $O_{A}$ and $O_{B}$. These orthogonal matrices translate into local unitary operations $U_{A}$ and $U_{B}$ on the quantum states, which, by definition, cannot change the entanglement properties. For further details see [1, 28].

[28] R. Horodecki, and P. Horodecki, Perfect correlations in the Einstein-Podolsky-Rosen experiment and Bell's inequalities, Phys. Lett. A 210, 227 (1996).

[29] Time-invariant ("frozen") discord has been previously observed in different systems [30-32].

[30] P. Haikka, T. H. Johnson, and S. Maniscalco, Non-Markovianity of local dephasing channels and time-invariant discord, Phys. Rev. A 87, 010103(R) (2013).

[31] F. M. Paula et al., Observation of Environment-Induced Double Sudden Transitions in Geometric Quantum Correlations, Phys. Rev. Lett. 111, 250401 (2013).

[32] B. Aaronson, R. Lo Franco, and G. Adesso, Comparative investigation of the freezing phenomena for quantum correlations under nondissipative decoherence, Phys. Rev. A 88, 012120 (2013).

[33] R. H. Dicke, Coherence in Spontaneous Radiation Processes, Phys. Rev. 93, 99 (1954).

[34] P. Lambropoulos, G. M. Nikolopoulos, T. R. Nielsen, and S. Bay, Fundamental quantum optics in structured reservoirs, Rep. Prog. Phys. 63, 455 (2000).

[35] H.-P. Breuer and F. Petruccione, The Theory of Open Quantum Systems (Oxford University Press, Oxford, UK, 2007).

[36] F. Levi and F. Mintert, Hierarchies of Multipartite Entanglement, Phys. Rev. Lett. 110, 150402 (2013).

[37] O. Gühne and M. Seevinck, Separability criteria for genuine multiparticle entanglement, New J. Phys. 12, 053002 (2010).

[38] M. Huber, F. Mintert, A. Gabriel, and B. C. Hiesmayr, Detection of High-Dimensional Genuine Multipartite Entanglement of Mixed States, Phys. Rev. Lett. 104, 210501 (2010).

[39] T. Gao, F. Yan, and S. J. van Enk, Permutationally Invariant Part of a Density Matrix and Nonseparability of N-Qubit States, Phys. Rev. Lett. 112, 180501 (2014).

[40] Here the density matrix is represented in the standard product basis of the eigenvectors of $\sigma_{z}$ [37].

[41] T. Monz et al., 14-Qubit Entanglement: Creation and Coherence, Phys. Rev. Lett. 106, 130506 (2011).

[42] H. Weyl, The Classical Groups (Princeton University, Princeton, NJ, 1946).

[43] M. Hayashi, Quantum Information (Springer, Berlin, Germany, 2006).

[44] H.-P. Breuer, E.-M. Laine, and J. Piilo, Measure for the Degree of Non-Markovian Behavior of Quantum Processes in Open Systems, Phys. Rev. Lett. 103, 210401 (2009). 


\section{Supplementary Material}

\section{TRACE PRESERVING PROPERTY OF THE COLLECTIVE DEPHASING MAP}

The operators $\Lambda_{ \pm}=\frac{1}{2}\left(\mathbb{I}_{2} \pm \boldsymbol{n} \cdot \boldsymbol{\sigma}\right)$ form a complete set of orthogonal projectors on the Hilbert space $\mathcal{H} \simeq \mathbb{C}^{2}$ of each qubit. These properties are inherited by the $\Theta_{i}$ operators, which are themselves orthogonal projectors: $\Theta_{i}=\Theta_{i}^{\dagger}$ and $\Theta_{i} \Theta_{j}=\Theta_{i} \delta_{i, j}$.

The trace preserving property of the map therefore reduces to $\sum_{i} \Theta_{i}^{\dagger} \Theta_{i}=\sum_{i} \Theta_{i}=\mathbb{I}_{2^{N}}$. This is simply proven by putting $t=0$ in Eq. (2) from the main text, and following the equalities from left to right:

$$
\sum_{i=0}^{N} \Theta_{i}=\left(\Lambda_{+}+\Lambda_{-}\right)^{\otimes N}=\mathbb{I}_{2^{N}}
$$

For the $A_{i}(t)$ operators we instead have

$$
\begin{aligned}
\sum_{i=0}^{N} A_{i}^{\dagger}(t) A_{i}(t) & =\sum_{i} \sum_{j, k} \lambda^{i}(t) \lambda_{j}^{i}(t)\left[\lambda_{k}^{i}(t)\right]^{*} \Theta_{i} \Theta_{j} \\
& =\sum_{i} \sum_{j} \lambda^{i}(t) \lambda_{j}^{i}(t)\left[\lambda_{j}^{i}(t)\right]^{*} \Theta_{j} \\
& =\sum_{j} \Theta_{j}=\mathbb{I}_{2^{N}},
\end{aligned}
$$

where we have used the spectral decomposition $M(t)=$ $\sum_{i} \lambda^{i}(t) \lambda^{i}(t) \lambda^{i^{\dagger}}(t)$, together with $M_{j j}(t)=1, \forall t$.

\section{CONSERVED TRACE OF THE $\beta$ MATRIX}

We now prove that the trace of the $\beta(t)$ matrix, defined by $\beta_{i j}(t)=\operatorname{tr}\left[\rho(t) \cdot \sigma_{i} \otimes \sigma_{j}\right]$, is a time-invariant quantity. From the definition we have

$$
\begin{aligned}
\operatorname{tr} \beta(t) & =\sum_{i=1}^{3} \beta_{i i}(t)=\sum_{i=1}^{3} \operatorname{tr}\left(\rho(t) \cdot \sigma_{i} \otimes \sigma_{i}\right) \\
& =\operatorname{tr}\left[\rho(t) \sum_{i=1}^{3} \sigma_{i} \otimes \sigma_{i}\right] .
\end{aligned}
$$

Notice now that the Bell state $\left|\Psi^{-}\right\rangle\left\langle\Psi^{-}\right|$reads [1]

$$
\left|\Psi^{-}\right\rangle\left\langle\Psi^{-}\right|=\frac{1}{4}\left(\mathbb{I}_{4}-\sum_{i=1}^{3} \sigma_{i} \otimes \sigma_{i}\right),
$$

which yields $\sum_{i} \sigma_{i} \otimes \sigma_{i}=\mathbb{I}_{4}-4\left|\Psi^{-}\right\rangle\left\langle\Psi^{-}\right|$. Substituting back we then have

$$
\begin{aligned}
\operatorname{tr} \beta(t) & =\operatorname{tr} \rho(t)-4 \operatorname{tr}\left[\rho(t)\left|\Psi^{-}\right\rangle\left\langle\Psi^{-}\right|\right] \\
& =1-4 \operatorname{tr}\left[\rho(t)\left|\Psi^{-}\right\rangle\left\langle\Psi^{-}\right|\right] .
\end{aligned}
$$

To prove that $\operatorname{tr} \beta(t)$ is conserved under time evolution, we compute its derivative and check whether it vanishes:

$$
\frac{\mathrm{d} \operatorname{tr} \beta(t)}{\mathrm{d} t}=-4 \operatorname{tr}\left[\dot{\rho}(t)\left|\Psi^{-}\right\rangle\left\langle\Psi^{-}\right|\right] .
$$

The time derivative of $\rho(t)$ reads

$$
\dot{\rho}(t)=\mathcal{L}_{t}[\rho(t)]=\sum_{i, j=0}^{N} \dot{M}_{i j}(t) \Theta_{i} \rho(t) \Theta_{j},
$$

which implies that

$$
\begin{aligned}
& \operatorname{tr}\left[\dot{\rho}(t)\left|\Psi^{-}\right\rangle\left\langle\Psi^{-}\right|\right] \\
& =\operatorname{tr}\left[\sum_{i, j=0}^{N} \dot{M}_{i j}(t) \Theta_{i} \rho(t) \Theta_{j}\left|\Psi^{-}\right\rangle\left\langle\Psi^{-}\right|\right] \\
& =\operatorname{tr}\left[\sum_{i, j=0}^{N} \dot{M}_{i j}(t) \Theta_{j}\left|\Psi^{-}\right\rangle\left\langle\Psi^{-}\right| \Theta_{i} \rho(t)\right] \\
& =\operatorname{tr}\left[\left(\mathcal{L}_{t}\left[\left|\Psi^{-}\right\rangle\left\langle\Psi^{-}\right|\right]\right)^{\dagger} \rho(t)\right] .
\end{aligned}
$$

However, the Bell state $\left|\Psi^{-}\right\rangle$is an eigenstate of the Hamiltonian of the system for whichever choice of $\boldsymbol{n}$, which means that it is itself unaffected by collective dephasing. This implies that $\mathcal{L}_{t}\left[\left|\Psi^{-}\right\rangle\left\langle\Psi^{-}\right|\right]=0, \forall t$ and therefore

$$
\frac{\mathrm{d}}{\mathrm{d} t} \operatorname{tr} \beta(t) \equiv 0 .
$$

\section{CONCURRENCE FOR BELL-DIAGONAL STATES}

In the tetrahedron of Bell-diagonal states [1], Wotters' concurrence [2] reads

$$
C(\boldsymbol{d})=\frac{1}{2} \max \left\{0,-1+\sum_{i}\left|d_{i}\right|\right\},
$$

where $\boldsymbol{d}=\left(d_{1}, d_{2}, d_{3}\right)^{T} \in \mathbb{R}^{3}$ is the point representing the quantum state (see main text).

For states in the $\left|\Psi_{-}\right\rangle$-corner, we have $d_{i} \leq 0, \forall i$. Hence, the concurrence in this corner can be rewritten as

$$
\begin{aligned}
C(\boldsymbol{d}) & =\frac{1}{2} \max \left\{0,-1-\sum_{i} d_{i}\right\} \\
& =\frac{1}{2} \max \{0,-1-k\},
\end{aligned}
$$

where we have used the fact that $\operatorname{tr} \beta=\sum_{i} d_{i}=k$ is a constant. This explicitly proves that Bell-diagonal states in this corner have time-invariant concurrence.

In the other corners of the tetrahedron, only one of the coordinates is negative. If we suppose that $d_{1} \leq 0$ (i.e. $\left|\Phi_{-}\right\rangle$corner), we have

$$
-1+\sum_{i}\left|d_{i}\right|=-1-d_{1}+d_{2}+d_{3}=-1+k-2 d_{1} .
$$


Let $\boldsymbol{d}$ represent the initial state, whereas $\boldsymbol{d}^{\mathrm{f}}$ represents the final, asymptotic state of the system after collective dephasing. Furthermore, we denote the negative components of $\boldsymbol{d}$ and $\boldsymbol{d}^{\mathrm{f}}$ with a subscript $j$, i.e., we have $d_{j} \leq 0$ and $d_{j}^{\mathrm{f}} \leq 0$, respectively. Direct application of the collective dephasing map leads to

$$
d_{j}^{\mathrm{f}}=\sum_{i} d_{i} n_{i}^{2}
$$

The concurrence in the final state is then

$$
\begin{aligned}
C\left(\boldsymbol{d}^{\mathrm{f}}\right) & =\frac{1}{2} \max \left\{0,-1+\sum_{i} d_{i}-2 \sum_{i} d_{i} n_{i}^{2}\right\} \\
& =\frac{1}{2} \max \left\{0,-1+\sum_{i}\left(1-2 n_{i}^{2}\right) d_{i}\right\} .
\end{aligned}
$$

From these formulae one can immediately notice that $C(d)=$ $C\left(\boldsymbol{d}^{\mathrm{f}}\right) \Longleftrightarrow \boldsymbol{n}= \pm \boldsymbol{e}_{j}$, where $\left\{\boldsymbol{e}_{1}, \boldsymbol{e}_{2}, \boldsymbol{e}_{3}\right\}$ is the standard basis of $\mathbb{R}^{3}$.

\section{TIME-INVARIANCE OF MULTIPARTITE WERNER STATES}

In this Section we prove that the multipartite Werner states [3] are time-invariant under the action of the collective dephasing map. Let $s \in \Sigma_{N}$ be a permutation in the symmetric group, and $V_{s}$ its representation in the operator space of $N$ qubits:

$$
V_{s}=\sum_{i_{1} \ldots i_{N} \in\{0,1\}}\left|i_{s(1)} \ldots i_{s(N)}\right\rangle\left\langle i_{1} \ldots i_{N}\right|
$$

The multipartite Werner states are then defined as $\rho_{W}=$ $\sum_{s \in \Sigma_{N}} c_{s} V_{s}$, where $c_{s}$ are arbitrary coefficients, leading to a valid quantum state $\rho_{W}$.
Analogously, if we define $k_{i}=i !(N-i)$ ! and $Q_{i}=\Lambda_{+}^{\otimes i} \otimes$ $\Lambda_{-}^{\otimes N-i}$, we can rewrite the $\Theta_{i}$ operators as

$$
\Theta_{i}=\frac{1}{k_{i}} \sum_{s \in \Sigma_{N}} V_{s} Q_{i} V_{s}^{\dagger}
$$

Direct application of the collective dephasing map yields

$$
\epsilon\left[\rho_{W}\right]=\sum_{i=0}^{N} \sum_{\pi, \sigma, \lambda \in \Sigma_{N}} \frac{c_{\pi}}{k_{i}^{2}}\left(V_{\sigma} Q_{i} V_{\sigma}^{\dagger}\right) V_{\pi}\left(V_{\lambda} Q_{i} V_{\lambda}^{\dagger}\right)
$$

Because $\Sigma_{N}$ is a closed group, the concatenation of two permutations describes another permutation, and therefore $\exists \alpha \in$ $\Sigma_{N}: V_{\sigma}=V_{\pi} V_{\alpha}$. The expression above can then be rewritten as

$$
\begin{aligned}
& \epsilon\left[\rho_{W}\right] \\
= & \sum_{i=0}^{N} \sum_{\pi, \alpha, \lambda \in \Sigma_{N}} \frac{c_{\pi}}{k_{i}^{2}} V_{\pi}\left(V_{\alpha} Q_{i} V_{\alpha}^{\dagger}\right)\left(V_{\lambda} Q_{i} V_{\lambda}^{\dagger}\right) \\
= & \sum_{\pi \in \Sigma_{N}} c_{\pi} V_{\pi} \sum_{i=0}^{N} \sum_{\alpha \in \Sigma_{N}} \frac{\left(V_{\alpha} Q_{i} V_{\alpha}^{\dagger}\right)}{k_{i}} \sum_{\lambda \in \Sigma_{N}} \frac{\left(V_{\lambda} Q_{i} V_{\lambda}^{\dagger}\right)}{k_{i}} \\
= & \sum_{\pi \in \Sigma_{N}} c_{\pi} V_{\pi} \sum_{i=0}^{N} \Theta_{i} \Theta_{i}=\rho_{W},
\end{aligned}
$$

where we have used the idempotency of the $\Theta_{i}$ operators and the closure relation $\sum_{i} \Theta_{i}=\mathbb{I}_{2^{N}}$.

*e.carnio@warwick.ac.uk

manuel.gessner@physik.uni-freiburg.de

[1] R. Horodecki, and M. Horodecki, Phys. Rev. A 54, 1838 (1996).

[2] W. K. Wootters, Phys. Rev. Lett. 80, 2245 (1998).

[3] T. Eggeling and R. F. Werner, Phys. Rev. A 63, 042111 (2001). 